\title{
The relationship between antenna loss and superdirectivity in MIMO systems
}

Michael A. Jensen

jensen@byu.edu

Nicolas W. Bikhazi

Follow this and additional works at: https://scholarsarchive.byu.edu/facpub

Part of the Electrical and Computer Engineering Commons

\section{Original Publication Citation}

Bikhazi, N. W., and M. A. Jensen. "The Relationship between Antenna Loss and Superdirectivity in MIMO Systems." Wireless Communications, IEEE Transactions on 6.5 (27): 1796-82

\section{BYU ScholarsArchive Citation}

Jensen, Michael A. and Bikhazi, Nicolas W., "The relationship between antenna loss and superdirectivity in MIMO systems" (2007). Faculty Publications. 259.

https://scholarsarchive.byu.edu/facpub/259 


\title{
The Relationship Between Antenna Loss and Superdirectivity in MIMO Systems
}

\author{
Nicolas W. Bikhazi and Michael A. Jensen, Senior Member, IEEE
}

\begin{abstract}
Multiple-input multiple-output (MIMO) communication systems can theoretically use array superdirectivity to optimally exploit the propagation channel. This superdirectivity leads to very large capacity bounds, particularly when the antennas are closely spaced. However, because superdirective behavior is difficult to achieve in practice, new capacity bounds have been formulated for the case where the level of array superdirectivity is constrained. Existing capacity analyses limit the superdirectivity in a suboptimal way. In this paper, the impact of superdirectivity in the capacity solution is limited by introducing finite ohmic loss in the transmit and receive antenna elements. Computational results reveal that even a small amount of ohmic loss significantly decreases the achievable system capacity.
\end{abstract}

Index Terms-Antenna gain, information theory, MIMO systems.

\section{INTRODUCTION}

$\mathbf{T}$ HE capacity gain achieved using multiple antennas at transmit and receive to communicate over multipath channels has been well-established [1]. Traditional analyses of these multiple-input multiple-output (MIMO) systems compute the channel capacity when the sum of the squares of the transmit excitation signals are constrained and the receiver noise is spatially white (i.e. has a diagonal covariance matrix) [2]. However, it has recently been demonstrated [3], [4] that if the radiated power is explicitly limited, transmit superdirectivity [5]-[9] solutions emerge which predict very high capacity increases. Similarly, when the external interference at the receiver is spatially colored, additional capacity gains appear due to the emergence of receiver superdirectivity solutions. While such gains are theoretically possible, achieving them is impractical given the difficulties associated with superdirectivity [6], [10]. Therefore, it is useful to formulate the capacity when the radiated power and the superdirectivity are constrained.

A prior analysis of this scenario has limited the impact of superdirectivity by constraining the transmit currents and receive beamformer weights [3], [4]. Specifically, these vector quantities are forced to lie in subspaces whose maximum superdirectivity $Q$ factor [7]-[9], which is approximately

Manuscript received August 16, 2005; revised August 4, 2006; accepted August 16, 2006. The associate editor coordinating the review of this paper and approving it for publication was R. Murch. This work was supported in part by the the National Science Foundation under Information Technology Grants CCR-0313056 and CCF-0428004 and in part by the U. S. Army Research Office under the Multi-University Research Initiative (MURI) Grant \# W911NF-04-1-0224.

The authors are with the Department of Electrical and Computer Engineering, Brigham Young University, 459 Clyde Building, Provo, UT 84602 USA (e-mail: nwb4@byu.edu; jensen@ee.byu.edu).

Digital Object Identifier 10.1109/TWC.2007.05633. inversely proportional to the achievable array communication bandwidth, is limited to lie below a predetermined level. However, as discussed in this work, this approach is not guaranteed to produce optimal capacity solutions and is somewhat difficult to tie to practical antenna parameters. In this paper, we propose to address the issue of limiting superdirectivity behavior in MIMO arrays by properly modeling antenna ohmic loss. At the transmitter, this loss creates considerable gain reduction for superdirective currents characterized by large magnitudes. At the receiver, ohmic loss leads to spatially white thermal noise which removes receiver superdirectivity. Unlike the formulation which constrains the maximum array $Q$ factor, this approach leads to optimal capacity solutions (under the stated assumptions) and is easily related to practical antenna properties. Representative computational examples illustrate the dramatic impact that even a small amount of loss can have on the system capacity.

\section{SUPERDIRECTIVITY IN MIMO SYSTEMS}

Demonstrating how antenna loss in a MIMO system removes superdirectivity requires development of a general communications model which can first reveal how the superdirectivity is produced. Throughout this paper boldface upper and lower case letters represent matrices (matrix $\mathbf{H}$ with $m$, $n$th element $H_{m n}$ ) and column vectors (vector $\mathbf{h}$ with $m$ th element $h_{m}$ ) respectively, $\{\cdot\}^{T}$ is a matrix transpose, and $\{\cdot\}^{\dagger}$ represents a matrix conjugate transpose. Furthermore, a narrowband channel is assumed so that all quantities are specified as phasors with an $e^{j \omega t}$ time dependence assumed and suppressed. We point out that the basic concepts presented here for suppressing superdirectivity could be extended to wideband analysis where the transmit and receive signal weights would be frequency dependent over the signal bandwidth.

\section{A. Communication Model}

Consider a MIMO communication system where $N_{\mathrm{t}}$ transmit antennas are linked to $N_{\mathrm{r}}$ receive antennas through a multipath propagation environment. The $n$th transmit and $m$ th receive elements have far-field vector radiation patterns for unit current excitations (with all adjacent elements terminated in an open-circuit) of $\mathbf{e}_{\mathrm{t} n}\left(\Omega_{\mathrm{t}}\right)$ and $\mathbf{e}_{\mathrm{r} m}\left(\Omega_{\mathrm{r}}\right)$ respectively, where $\Omega_{\mathrm{t}}$ and $\Omega_{\mathrm{r}}$ represent solid angle coordinates referenced to the transmit and receive coordinate origins, respectively, and the pattern vector elements represent the possible pattern polarizations. Because the patterns are computed with other elements open-circuited, the radiation pattern at any instant is the superposition of these patterns weighted by the current 
on each element, and we therefore let $\mathrm{x}$ represent the $N_{\mathrm{t}} \times 1$ vector of current inputs to the transmit array. Assuming all scatterers lie in the array far-field, the $N_{\mathrm{r}} \times 1$ open-circuit received signal voltage vector can be expressed as [4]

$$
\begin{aligned}
\mathbf{v}_{\mathrm{s}} & =\mathbf{H x} \\
H_{m n} & =\int d \Omega_{\mathrm{r}} \int d \Omega_{\mathrm{t}} \mathbf{e}_{\mathrm{r} m}^{T}\left(\Omega_{\mathrm{r}}\right) \mathbf{G}\left(\Omega_{\mathrm{r}}, \Omega_{\mathrm{t}}\right) \mathbf{e}_{\mathrm{t} n}\left(\Omega_{\mathrm{t}}\right),
\end{aligned}
$$

where the integrals are over a full $4 \pi$ steradians and $\mathbf{G}\left(\Omega_{\mathrm{r}}, \Omega_{\mathrm{t}}\right)$ is a dyadic Green's function with element $G_{i j}$ relating the received field in the $i$ th receive polarization to the transmitted field in the $j$ th polarization.

Assume also that an external vector interference field (using the same polarization description as used for the radiation patterns) denoted by $\psi\left(\Omega_{\mathrm{r}}\right)$ impinges on the receive array. This field is modeled as a zero-mean complex Gaussian random vector, with the field arriving at angle $\Omega_{\mathrm{r}}$ uncorrelated with that arriving at angle $\Omega_{\mathrm{r}}^{\prime} \neq \Omega_{\mathrm{r}}$ and with field in one polarization uncorrelated with that in another. Mathematically, this is expressed as

$$
\mathrm{E}\left\{\boldsymbol{\psi}\left(\Omega_{\mathrm{r}}\right) \boldsymbol{\psi}^{\dagger}\left(\Omega_{\mathrm{r}}^{\prime}\right)\right\}=\sigma_{\mathrm{i}}^{2} p_{\mathrm{i}}\left(\Omega_{\mathrm{r}}\right) \delta\left(\Omega_{\mathrm{r}}-\Omega_{\mathrm{r}}^{\prime}\right) \mathbf{I},
$$

where $\mathrm{E}\{\cdot\}$ is an expectation, $\mathbf{I}$ is the identity matrix, and $\delta(\cdot)$ is the Dirac delta function. The function $\sigma_{\mathrm{i}}^{2} p_{\mathrm{i}}\left(\Omega_{r}\right)$ represents the interference angular power spectrum, where $\sigma_{\mathrm{i}}^{2}$ specifies the interference power level and $p_{\mathrm{i}}\left(\Omega_{\mathrm{r}}\right)$ has unit area. Constructing the matrix $\mathbf{E}_{\mathrm{r}}\left(\Omega_{\mathrm{r}}\right)$ with $m$ th column $\mathbf{e}_{\mathrm{r} m}\left(\Omega_{\mathrm{r}}\right)$, the vector of received interference voltages becomes

$$
\mathbf{v}_{\mathrm{i}}=\int d \Omega_{\mathrm{r}} \mathbf{E}_{\mathrm{r}}^{T}\left(\Omega_{\mathrm{r}}\right) \boldsymbol{\psi}\left(\Omega_{\mathrm{t}}\right)
$$

whose covariance can be constructed using (3) as

$$
\mathbf{K}_{\mathrm{i}}=\mathrm{E}\left\{\mathbf{v}_{\mathrm{i}} \mathbf{v}_{\mathrm{i}}^{\dagger}\right\}=\sigma_{\mathrm{i}}^{2} \int d \Omega_{\mathrm{r}} \mathbf{E}_{\mathrm{r}}^{T}\left(\Omega_{\mathrm{r}}\right) \mathbf{E}_{\mathrm{r}}^{*}\left(\Omega_{\mathrm{r}}\right) p_{\mathrm{i}}\left(\Omega_{\mathrm{r}}\right)
$$

The total open-circuit voltage on the receive elements becomes

$$
\mathbf{y}=\mathbf{H x}+\mathbf{v}_{\mathrm{i}} .
$$

It is important to recognize that this analysis considers only the open-circuit voltages rather than the voltages across an actual set of receiver terminations. Modeling such a receiver front-end will alter the system by linearly transforming the signal and interference vectors and by adding a contribution of thermal noise from the front-end amplifiers [11]. In this analysis, however, amplifier noise is neglected since receiver superdirectivity occurs in the presence of spatially colored external interference as opposed to the spatially white internal thermal noise. When this amplifier noise is neglected the linear transformation of the signal and interference created by the front-end network will not alter the system capacity. Therefore, analysis using the open-circuit voltages is adequate for assessing the behavior of arrays when superdirectivity is considered.

\section{B. Capacity and Superdirectivity}

In traditional analyses of MIMO systems, the average sum of the squares of the excitation current magnitudes is constrained in the capacity formulation. When the elements of the transmit array have reasonable inter-element spacings (greater than $\lambda / 2$, where $\lambda$ is the free-space wavelength), this quantity is approximately proportional to the power radiated. However, for closely spaced elements, the capacity solution can lead to large levels of radiated power [4] which naturally leads to artificially high capacity levels. The goal of the analysis in this section is therefore to determine the capacity when the radiated power is explicitly limited, although this constraint will be modified slightly in Section III.

For a transmit array with a reciprocal impedance matrix $\mathbf{Z}_{t}$, the average power radiated by the array for a vector $\mathbf{x}$ of transmit currents is given by [4]

$$
P_{\text {rad }}=\frac{1}{2} \mathrm{E}\left\{\mathbf{x}^{\dagger} \operatorname{Re}\left[\mathbf{Z}_{\mathrm{t}}\right] \mathbf{x}\right\}=\operatorname{Tr}\left\{\mathbf{K}_{x} \mathbf{R}_{\mathrm{t}}\right\},
$$

where $\operatorname{Re}[\cdot]$ is the real part, $\mathbf{K}_{x}$ is the covariance of $\mathbf{x}$, and $\operatorname{Tr}\{\cdot\}$ is a trace. The matrix $\mathbf{R}_{\mathrm{t}}=\operatorname{Re}\left[\mathbf{Z}_{\mathrm{t}}\right] / 2$ represents an effective radiation resistance for the array (scaled by a factor of $1 / 2$ ) which can be computed either using an impedance computation or from

$$
\mathbf{R}_{\mathrm{t}}=\frac{1}{2 Z_{0}} \int d \Omega_{\mathrm{t}} \mathbf{E}_{\mathrm{t}}^{\dagger}\left(\Omega_{\mathrm{t}}\right) \mathbf{E}_{\mathrm{t}}\left(\Omega_{\mathrm{t}}\right),
$$

where $Z_{0}$ is the free-space intrinsic impedance and $\mathbf{E}_{\mathrm{t}}\left(\Omega_{\mathrm{t}}\right)$ is the matrix with $n$th column $\mathbf{e}_{\mathrm{t} n}\left(\Omega_{\mathrm{t}}\right)$.

If we now restrict the radiated power such that $P_{\mathrm{rad}} \leq P_{T}$, where $P_{T}$ is a set threshold, then the mutual information of the MIMO system described by (6) is given by

$$
\begin{aligned}
I(\mathbf{y} ; \mathbf{x}) & \leq \log _{2} \operatorname{det}\left(\widetilde{\mathbf{H}} \widetilde{\mathbf{K}}_{x} \widetilde{\mathbf{H}}^{\dagger}+\mathbf{I}\right) \\
\widetilde{\mathbf{H}} & =\mathbf{K}_{\mathrm{i}}^{-1 / 2} \mathbf{H R}_{\mathrm{t}}^{-1 / 2} \\
\widetilde{\mathbf{K}}_{x} & =\mathbf{R}_{\mathrm{t}}^{1 / 2} \mathbf{K}_{x} \mathbf{R}_{\mathrm{t}}^{1 / 2},
\end{aligned}
$$

with the constraint $\operatorname{Tr}\left\{\widetilde{\mathbf{K}}_{x}\right\} \leq P_{T}$. The covariance $\widetilde{\mathbf{K}}_{x}$ can be determined from the water-filling solution to construct the capacity bound [2], [12].

It has been recently shown that the solution to this modified capacity formulation exhibits interesting behavior when the antennas become closely spaced [3], [4]. Traditional understanding is that under such circumstances, the resulting increased signal correlation results in reduced channel capacity. However, it is also known that very compact arrays can be excited such that they achieve superdirective solutions [5]-[9]. Under this case, the spatial filtering capability of the array is enhanced, which leads to increased capacity [4]. It is important to recognize that such superdirectivity is not observed in most capacity formulations since the traditional constraint limits the transmit current magnitudes, while superdirective solutions require large currents to achieve reasonable radiated power levels. However, under the radiated power constraint the superdirective solutions are favorable since they result in high channel gain with modest radiated power [4].

Mathematically, superdirectivity for compact arrays is manifest by the small eigenvalues of the matrices $\mathbf{R}_{\mathrm{t}}$ and $\mathbf{K}_{\mathrm{i}}$ which create high channel gain without requiring large amounts of radiated power. At the transmitter, this phenomenon can be related to array superdirectivity by first defining $\mathbf{A}_{\mathrm{t}}=$ $\mathbf{R}_{\mathrm{t}} / R_{\mathrm{t}, 11}$ so that the superdirectivity $Q$ factor (which relates 
to the achievable system bandwidth) is [7], [8]

$$
Q_{\mathrm{t}}=\frac{\mathbf{x}^{\dagger} \mathbf{x}}{\mathbf{x}^{\dagger} \mathbf{A}_{\mathrm{t}} \mathbf{x}} \text {. }
$$

The small eigenvalues of $\mathbf{R}_{t}$ associated with high channel gain are scaled versions of the small eigenvalues of $\mathbf{A}_{\mathrm{t}}$ and are associated with large array $Q$ factors indicative of superdirectivity. A similar tie can be made for the receiver to show that the small eigenvalues of $\mathbf{K}_{\mathrm{i}}$ are associated with array superdirectivity.

It has also been recently demonstrated that this superdirectivity can be limited by passing the transmit current and received voltage vectors through beamformers restricting the excitations to a non-superdirective space [3], [4]. The nonsuperdirective space is formed from the eigenvectors of $\mathbf{A}_{\mathrm{t}}$ (or a receiver equivalent) whose eigenvalues ensure that the $Q$ factor of the array will be below a specified level. However, this solution is not optimal. Specifically, it is possible to form currents from a linear combintation of the vectors from the superdirective and non-superdirective spaces that achieve an overall $Q$ factor that is below the given threshold. Stated another way, the beamformer limits the excitation currents or receive weights to lie in a subspace, while the actual constraint should limit these vectors to an ellipsoid in the multi-dimensional space. Unfortunately, there does not appear to be an obvious way to achieve the optimal solution using the beamforming approach combined with the capacity solution.

\section{AnTENNA OHMic Loss}

Given the limitations of the beamforming approach for determining system capacity when superdirectivity is constrained, we instead propose to accomplish this computation by properly including the antenna ohmic loss in the channel model. Because transmit superdirectivity solutions are characterized by high current magnitudes, the loss introduced by even a small antenna resistance will make superdirective excitations inefficient and unfavorable relative to nonsuperdirective ones. At the receiver, the ohmic loss leads to spatially white thermal noise that will remove receive superdirective solutions. Because of the difference in the effects at the transmitter and receiver, we develop the impact of antenna loss at each end of the link separately.

\section{A. Transmitter}

Incorporating transmit antenna loss as part of the channel implies that the capacity formulation power constraint must limit the power delivered to the transmit array rather than the power radiated, since some of the power will be consumed by antenna loss. We will formulate the capacity under this delivered constraint in this section and then discuss an approach for compensating for the reduction in radiated power in Section III-C. To implement the power constraint, we first construct the average power delivered to the antenna array using

$$
P_{\text {in }}=\mathrm{E}\left\{\mathbf{x}^{\dagger}\left(\mathbf{R}_{\mathrm{t}}+\mathbf{L}_{\mathrm{t}}\right) \mathbf{x}\right\}=\operatorname{Tr}\left\{\mathbf{K}_{x} \widehat{\mathbf{R}}_{\mathrm{t}}\right\} .
$$

The $n$th element of the diagonal matrix $\mathbf{L}_{\mathrm{t}}$ represents onehalf the antenna ohmic loss resistance for the $n$th antenna. We emphasize that this is the physical antenna resistance, which can be obtained from radiation efficiency measurements for practical scenarios [13]. For arrays constructed of identical elements, this matrix will be $\mathbf{L}_{\mathrm{t}}=L_{\mathrm{t}} \mathbf{I}=\left(R_{\mathrm{t}, \text { loss }} / 2\right) \mathbf{I}$, where $R_{\mathrm{t}, \text { loss }}$ is the loss resistance of each element.

If the delivered power is constrained in the capacity formulation, then $\widehat{\mathbf{R}}_{\mathrm{t}}$ will replace $\mathbf{R}_{\mathrm{t}}$ in (10) and (11). The addition of the diagonal matrix $\mathbf{L}_{\mathrm{t}}$ in $\widehat{\mathbf{R}}_{\mathrm{t}}$ will eliminate the very small eigenvalues associated with superdirectivity and therefore regularize the matrix inverse $\widehat{\mathbf{R}}_{\mathrm{t}}^{-1 / 2}$ even when the antenna loss is modest. This is a mathematical indication of the fact that superdirective solutions will exhibit high loss and become unfavorable relative to more traditional excitations.

For the example computations that follow, all array elements will be assumed identical so that $Z_{\mathrm{t}, n n}$ will be the same for all $n$. We can therefore rearrange $\widehat{\mathbf{R}}_{\mathrm{t}}$ as

$$
\begin{aligned}
\widehat{\mathbf{R}}_{\mathrm{t}} & =R_{\mathrm{t}, 11}\left[\mathbf{A}_{\mathrm{t}}+\frac{L_{\mathrm{t}}}{R_{\mathrm{t}, 11}} \mathbf{I}\right] \\
& =R_{\mathrm{t}, 11}\left[\mathbf{A}_{\mathrm{t}}+\left(1 / k_{a}-1\right) \mathbf{I}\right],
\end{aligned}
$$

where $k_{a}=R_{\mathrm{t}, 11} /\left(R_{\mathrm{t}, 11}+L_{\mathrm{t}}\right)$ is the single element efficiency [13]. This will allow demonstration of the impact of loss on superdirectivity as a function of this practical efficiency parameter.

\section{B. Receiver}

At the receiver, ohmic loss does not explicitly change the possibility of observing receive superdirectivity in the capacity solution since the loss will operate identically on the signal and the external interference. However, in this case the resistance adds a thermal noise component to the received signal that must be modeled correctly. Specifically, if the receive array is characterized by a diagonal ohmic loss resistance matrix $\mathbf{L}_{\mathrm{r}}$, then an open-circuit noise voltage vector $\boldsymbol{\eta}$ will be introduced so that the received signal becomes

$$
\mathbf{y}^{\prime}=\mathbf{H x}+\mathbf{v}_{\mathrm{i}}+\boldsymbol{\eta} .
$$

Since the noise on each antenna is assumed independent of the noise on all other antennas, the covariance of this noise will be [14]

$$
\mathbf{K}_{\eta}=\mathrm{E}\left\{\boldsymbol{\eta} \boldsymbol{\eta}^{\dagger}\right\}=4 k_{B} T \mathbf{L}_{\mathrm{r}}
$$

where $k_{B}$ is the Boltzmann constant and $T$ is the temperature. Note that this expression does not contain the commonly used system power bandwidth because in this narrowband phasor analysis all covariances represent power spectral densities which are normalized by the bandwidth.

Given this spatially white thermal noise contribution, which is assumed independent of the external interference, the total interference plus noise has covariance

$$
\widehat{\mathbf{K}}_{\eta}=\mathbf{K}_{\mathrm{i}}+\mathbf{K}_{\eta}
$$

The addition of the diagonal matrix $\mathbf{K}_{\eta}$ provides the regularization required to avoid receive superdirectivity. Once again, for the computations shown in this paper, all array elements 


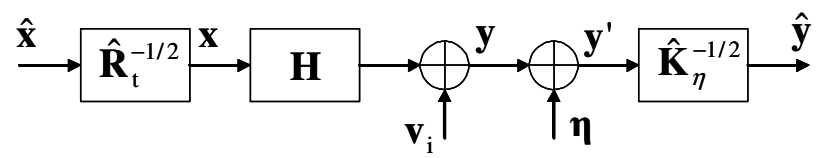

Fig. 1. Equivalent model for the system whose capacity is described by (19).

will be assumed identical so that $K_{\mathrm{i}, m m}$ will be the same for all $m$ and $\mathbf{L}_{\mathrm{r}}=L_{\mathrm{r}} \mathbf{I}$. We can therefore write

$$
\widehat{\mathbf{K}}_{\eta}=K_{\mathrm{i}, 11}\left[\mathbf{B}_{\mathrm{r}}+\frac{K_{\eta, 11}}{K_{\mathrm{i}, 11}} \mathbf{I}\right]=K_{\mathrm{i}, 11}\left[\mathbf{B}_{\mathrm{r}}+\frac{1}{\mathrm{INR}} \mathbf{I}\right] \text {, }
$$

where $\mathbf{B}_{\mathrm{r}}=\mathbf{K}_{\mathrm{i}} / K_{\mathrm{i}, 11}$ and INR is the interference-to-noise ratio.

\section{Capacity}

With the antenna ohmic loss now properly included, the mutual information for the model in (15) becomes

$$
\begin{aligned}
I\left(\mathbf{y}^{\prime} ; \mathbf{x}\right) & \leq \log _{2} \operatorname{det}\left(\widehat{\mathbf{H}} \widehat{\mathbf{K}}_{x} \widehat{\mathbf{H}}^{\dagger}+\mathbf{I}\right) \\
\widehat{\mathbf{H}} & =\widehat{\mathbf{K}}_{\eta}^{-1 / 2} \mathbf{H} \widehat{\mathbf{R}}_{\mathrm{t}}^{-1 / 2} \\
\widehat{\mathbf{K}}_{x} & =\widehat{\mathbf{R}}_{\mathrm{t}}^{1 / 2} \mathbf{K}_{x} \widehat{\mathbf{R}}_{\mathrm{t}}^{1 / 2},
\end{aligned}
$$

with the constraint $\operatorname{Tr}\left\{\widehat{\mathbf{K}}_{x}\right\} \leq P_{T}$. The covariance $\widehat{\mathbf{K}}_{x}$ can now be determined from the water-filling solution. Because the antenna loss will result in reduced radiated power, we can compute $\mathbf{K}_{x}$ from (21) and then scale it as $\overline{\mathbf{K}}_{x}=\alpha \mathbf{K}_{x}$ so that $P_{\text {rad }}=\operatorname{Tr}\left\{\overline{\mathbf{K}}_{x} \mathbf{R}_{\mathrm{t}}\right\}=P_{T}$. Using $\overline{\mathbf{K}}_{x}$ in place of $\mathbf{K}_{x}$ when evaluating (19) then provides the capacity bound under the delivered power constraint (to suppress transmit superdirectivity) but with the impact of the reduced radiated power removed. If the transmitter has no knowledge of the propagation channel represented by $\mathbf{H}$, then the uninformed transmitter capacity can be computed by repeating this procedure with $\widehat{\mathbf{K}}_{x}=\left(P_{T} / N_{\mathrm{t}}\right) \mathbf{I}$.

It is possible to interpret this revised mutual information expression in terms of the equivalent communication system shown in Fig. 1. Specifically, the effective input vector $\widehat{\mathbf{x}}$ is fed into a beamforming network so that $\mathbf{x}=\widehat{\mathbf{R}}_{\mathrm{t}}^{-1 / 2} \widehat{\mathbf{x}}$. This vector is sent over the channel and is augmented by the additive interference and the thermal noise created by the receive antenna loss to create $\mathbf{y}^{\prime}$. Finally, the rearrangement of the noise covariance within the mutual information expression of (19) is equivalent to creating a noise pre-whitening beamformer so that $\widehat{\mathbf{y}}=\widehat{\mathbf{K}}_{\eta}^{-1 / 2} \mathbf{y}^{\prime}$.

\section{Simulation Examples}

Computational examples serve to demonstrate the application of the approach considered here to real scenarios as well as to illustrate that even very small amounts of ohmic loss can significantly remove the impact of superdirectivity on capacity.

\section{A. Simulation Assumptions}

Unless otherwise noted, the transmitting and receiving antennas are uniform circular arrays (UCAs) of 16 infinitesimal dipole elements [13] with a circle diameter of $D$ specified in

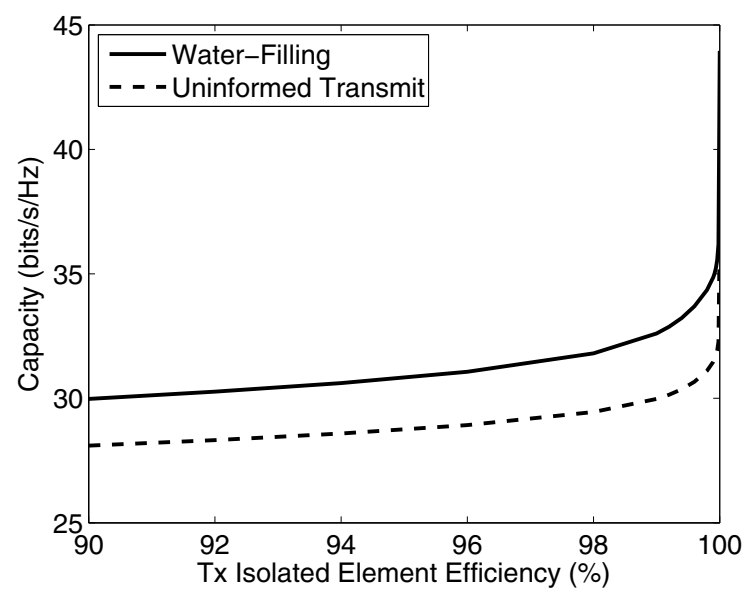

Fig. 2. Water-filling and uninformed transmit capacity versus transmit isolated element efficiency for INR $=10 \mathrm{~dB}$ and 16-element UCAs with diameter $D=0.5 \lambda$.

free-space wavelengths $(\lambda)$. Also, the external interference is confined to the horizontal plane with a uniform angular power spectrum, or

$$
p_{\mathrm{i}}\left(\Omega_{\mathrm{r}}\right)=\frac{1}{2 \pi} \delta\left(\theta_{\mathrm{r}}-\pi / 2\right),
$$

where $\theta_{\mathrm{r}}$ represents the receive elevation angle. Under these conditions, $\mathbf{K}_{\mathrm{i}}$ and $\mathbf{R}_{\mathrm{t}}$ are computed from (5) and (8), respectively.

The narrowband propagation channel is represented using a path-based statistical model where the departures and arrivals are clustered in angle. Here again, signal propagation is confined to the horizontal plane. The discussion in [15] provides a description of the model, including the parameters used here which are representative of an indoor environment. For all computations, 500 random realizations of this propagation model and the corresponding channel transfer function $\mathbf{H}_{0}$ are generated, and each matrix is normalized according to

$$
\mathbf{H}=\frac{\sqrt{N_{\mathrm{r}} N_{\mathrm{t}}}}{\left\|\mathbf{H}_{0}\right\|_{F}} \mathbf{H}_{0},
$$

where $\|\cdot\|_{F}$ is the Frobenius matrix norm. This normalization ensures that all channel realizations achieve the same average signal-to-interference ratio (SIR), and therefore removes the impact of channel path loss or shadowing on the results. Then, for single isolated transmit and receive dipoles, the scalar values of $K_{\mathrm{i}}$ and $R_{\mathrm{t}}$ are computed from (5) and (8), respectively. Under these conditions, the single-input singleoutput (SISO) SIR can be defined as

$$
\mathrm{SIR}=\frac{P_{T}}{R_{\mathrm{t}} K_{\mathrm{i}}}
$$

For all computations that follow, the SISO SIR is set to $20 \mathrm{~dB}$.

\section{B. Capacity}

Fig. 2 shows the water-filling and uninformed transmit capacity as a function of the radiation efficiency $\left(k_{a}\right.$ in (14)) of the transmit elements in isolation for an interference-to-noise ratio (INR) of $10 \mathrm{~dB}$ and UCA diameter $D=\lambda / 2$. Most apparent is the fact that the optimal water-filling capacity is 


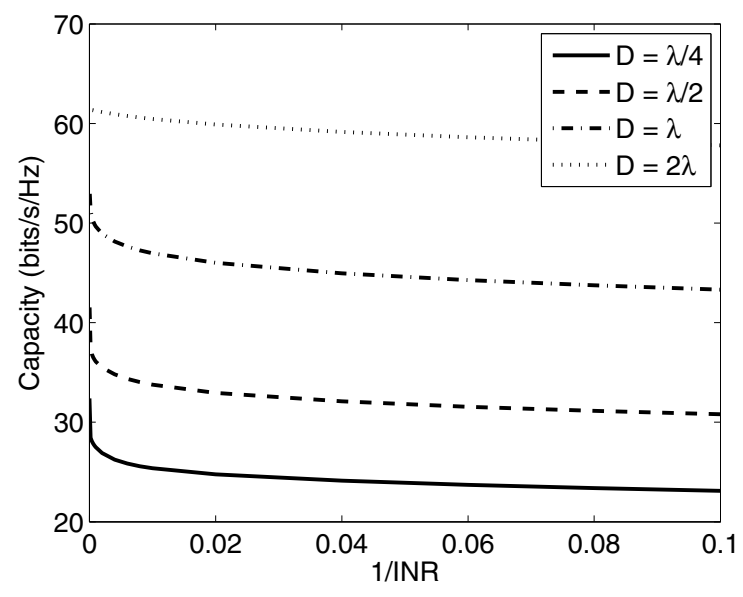

Fig. 3. Water-filling capacity versus INR for a transmit element efficiency of $95 \%$ and four different radii for the 16-element UCAs.

somewhat larger than the corresponding value obtained using the suboptimal uninformed transmitter solution. Otherwise, the curves show similar trends implying that the same physical phenomena apply to both capacity solutions. These curves reveal that as the antenna efficiency is increased, the capacity increases, with the most dramatic impact occuring around $99 \%$ efficiency where superdirective excitations dominate the solution. It is noteworthy that the $99 \%$ threshold efficiency is very difficult to achieve in practice, suggesting that true transmit superdirective behavior would not be observed in a practical system.

Fig. 3 shows the water-filling capacity as a function of the INR for an isolated transmit element efficiency of $95 \%$ and four different UCA diameters (antenna spacing reduces with diameter). The horizontal axis is actually expressed as 1/INR to emphasize the dramatic change in capacity as the thermal noise goes to zero (antenna becomes lossless). For small arrays, the close element spacing enables superdirectivity which accounts for the sharp capacity increase for small antenna loss. The performance of the large array, on the other hand, is dominated by the thermal noise level because the increased element spacing does not allow superdirective solutions.

The computational simplicity afforded by the assumption of infinitesimal dipoles allows very efficient analysis of the impact of antenna ohmic loss on array superdirective behavior. However, for these antennas the radiation pattern of the driven element with all other elements open-circuited (as specified in Section II-A) is the same as the pattern for the element in isolation. For practical antennas, this open-circuit radiation pattern differs from the isolated element radiation pattern, which could potentially alter the array superdirective behavior.

To study this effect, we repeat the computation considered in Fig. 2 using half-wave dipoles in place of the infinitesimal dipoles. The open-circuit radiation patterns and impedance matrix are computed for each array configuration using the freely-available Numerical Electromagnetics Code (NEC) [16] assuming a dipole wire radius of $0.005 \lambda$. Fig. 4 shows the outcome of this computation. The key difference between these results for practical dipoles and those provided in Fig. 2 for ideal (infinitesimal) elements is the absolute capacity level.

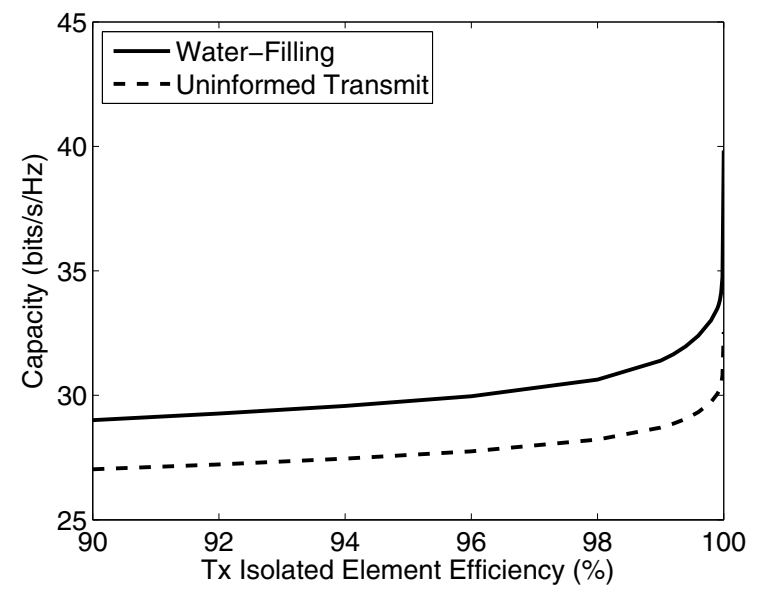

Fig. 4. Water-filling and uninformed transmit capacity versus transmit isolated element efficiency for INR $=10 \mathrm{~dB}$ and 16-element UCAs with diameter $D=0.5 \lambda$ and half-wave dipole elements.

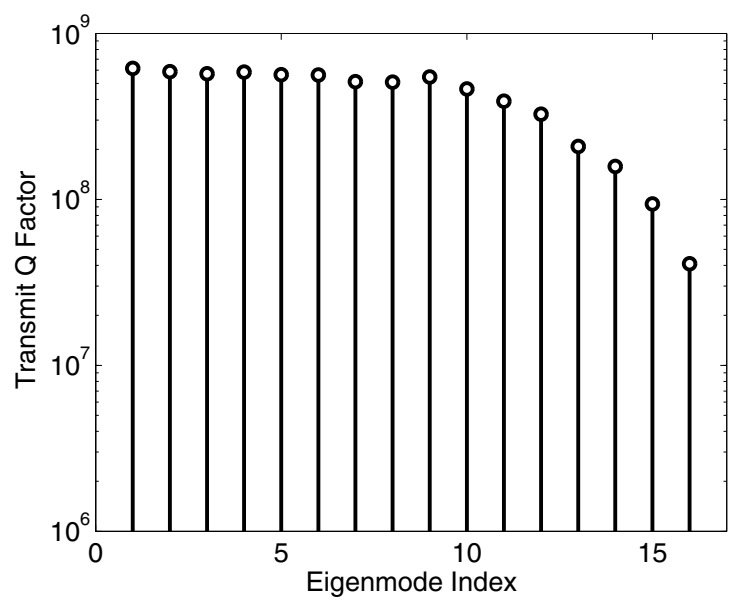

Fig. 5. $Q$ factor for each of the channel eigenmodes for a transmit element efficiency of $100 \%$, INR $=10 \mathrm{~dB}$, and a 16-element UCA with diameter $D=0.5 \lambda$.

Otherwise, the strong similarity between these two sets of results suggests that superdirective behavior remains a possibility despite the pattern distortion created by the coupling.

\section{Quantification of Superdirectivity}

It is useful to examine the level of superdirectivity in the capacity solutions. Unfortunately, measuring superdirectivity using quantities such as the array $Q$ factor in (12) requires evaluating the properties of specific transmit excitations, while the capacity only specifies the excitation covariance. However, since the water-filling solution constructs the transmit covariance using the basis of the right singular vectors of the channel matrix, examination of (19) and (21) reveals that in our case, the columns of the matrix $\widehat{\mathbf{R}}_{\mathrm{t}}^{-1 / 2} \widehat{\mathbf{V}}$, where $\widehat{\mathbf{V}}$ represents the right singular vectors of $\widehat{\mathbf{H}}$, form the basis for the transmit covariance $\mathbf{K}_{x}$. We can therefore interpret each such column in turn as the vector $\mathbf{x}$ in (12) to obtain a $Q$ factor for each channel eigenmode. Fig. 5 provides the value of this $Q$ factor averaged over the 500 channel realizations for the 16-element UCA of diameter $D=0.5 \lambda$ with $100 \%$ transmit 
element efficiency and an INR of $10 \mathrm{~dB}$. As can be seen, the $Q$ factors of the dominant modes are very large. This implies that even if a simple beamforming solution is used (rather than the optimal solution) where only the dominant channel eigenmode is exploited for communication, transmit superdirectivity will play a role in determining system performance.

To obtain a more inclusive measure of the array superdirectivity obtained from the capacity formulation, we can define an effective $Q$ factor for the communication. At the transmitter, we take the expectation of the numerator and the denominator of (12) individually, or

$$
\bar{Q}_{\mathrm{t}}=\frac{\mathrm{E}\left\{\mathbf{x}^{\dagger} \mathbf{x}\right\}}{\mathrm{E}\left\{\mathbf{x}^{\dagger} \mathbf{A}_{\mathrm{t}} \mathbf{x}\right\}}=\frac{\operatorname{Tr}\left\{\mathbf{K}_{x}\right\}}{\operatorname{Tr}\left\{\mathbf{K}_{x} \mathbf{A}_{\mathrm{t}}\right\}} .
$$

Since the capacity solution provides a value of $\mathbf{K}_{x}$, this expression can be computed for each channel realization.

At the receiver, the discussion in Section III-C along with the equivalent system model in Fig. 1 suggest that the vector given by

$$
\widehat{\mathbf{s}}=\widehat{\mathbf{K}}_{\eta}^{-1 / 2} \mathbf{H} \mathbf{x}=\mathbf{H}_{\mathrm{r}} \mathbf{x}
$$

represents the (noiseless) signal at the output of the noise prewhitening beamformer and has covariance

$$
\widehat{\mathbf{K}}_{s}=\mathbf{H}_{\mathrm{r}} \mathbf{K}_{x} \mathbf{H}_{\mathrm{r}}^{\dagger} \text {. }
$$

To define an effective $Q$ factor for this receiver, we assume the receive beamformer is reciprocal. Then we consider $\widehat{\mathbf{s}}$ as a transmit vector that is to be passed back through the receive beamformer, yielding an effective transmit signal at the antenna terminals of $\mathbf{s}=\widehat{\mathbf{K}}_{\eta}^{-1 / 2} \widehat{\mathbf{s}}$ with covariance

$$
\mathbf{K}_{s}=\widehat{\mathbf{K}}_{\eta}^{-1 / 2} \widehat{\mathbf{K}}_{s} \widehat{\mathbf{K}}_{\eta}^{-1 / 2} \text {. }
$$

The effective receive $Q$ factor is given by

$$
\bar{Q}_{\mathrm{r}}=\frac{\operatorname{Tr}\left\{\mathbf{K}_{s}\right\}}{\operatorname{Tr}\left\{\mathbf{K}_{s} \mathbf{A}_{\mathrm{r}}\right\}},
$$

where $\mathbf{A}_{\mathrm{r}}$ is the receive array counterpart to $\mathbf{A}_{\mathrm{t}}$.

In the computations, the value of the effective $Q$ factor is averaged over the 500 channel realizations. Fig. 6 plots the effective transmit $Q$ factor as a function of transmit element efficiency for the same conditions as considered in Fig. 2. It can be seen in Fig. 6 that as the antenna efficiency goes to $100 \%$ this average $Q$ factor achieves very high values indicative of superdirectivity behavior. Fig. 7 plots the effective receive $Q$ factor as a function of INR for the same conditions as considered in Fig. 3 for two array sizes. Once again, these results confirm that for the small array spacing, the increase in capacity with increasing INR is a result of signals with high $Q$ factors indicative of receive superdirectivity.

\section{CONCLUSiON}

This paper has formulated the mutual information for a MIMO system under the conditions where the power input to the transmit array is constrained, the receiver is subject to external interference, and antenna ohmic loss is properly modeled. The results show that as the antenna loss is reduced to zero, superdirective solutions to the capacity formulation

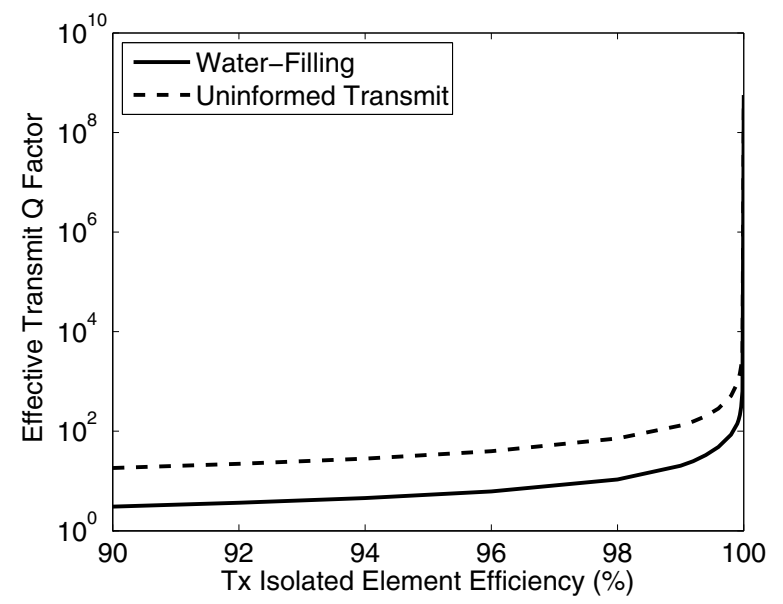

Fig. 6. Effective transmit $Q$ factor versus transmit isolated element efficiency for $\mathrm{INR}=10 \mathrm{~dB}$ and 16-element UCAs with diameter $D=0.5 \lambda$. Results for water-filling and uninformed transmit capacity solutions are provided.

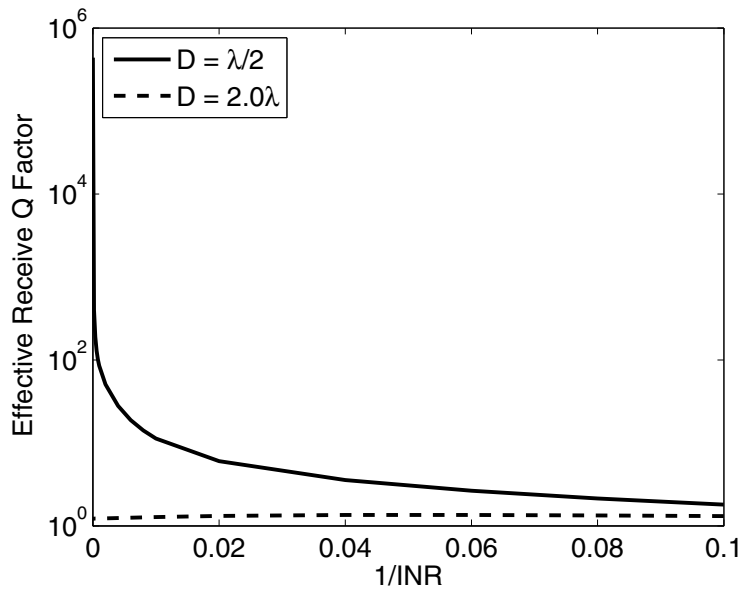

Fig. 7. Effective receive $Q$ factor versus INR for a transmit element efficiency of $95 \%$ and two different radii for the 16-element UCAs. Results are for the water-filling capacity solution.

emerge. However, introduction of antenna ohmic loss can dramatically reduce the impact of superdirectivity on the capacity results. Computational examples illustrate the application of the method and reveal that this superdirectivity is virtually eliminated when only a small amount of ohmic loss is present.

While this formulation has chosen to use antenna ohmic loss as a practical parameter to include in the model, other imperfections such as loss in feeding lines and matching networks or spatially white noise introduced in front-end amplifiers will similarly remove superdirective solutions provided that these impairments are included in the channel model. Therefore, this approach, coupled with expanded network and noise analysis techniques, provides a comprehensive framework for modeling the capacity behavior of closely spaced antenna elements.

\section{REFERENCES}

[1] G. J. Foschini and M. J. Gans, "On limits of wireless communications in a fading environment when using multiple antennas," Wireless Pers. Commun., vol. 6, pp. 311-335, Mar. 1998. 
[2] M. A. Khalighi, J. Brossier, G. Jourdain, and K. Raoof, "Water filling capacity of Rayleigh MIMO channels," in Proc. IEEE 12th Intl. Symp. Personal, Indoor Mobile Radio Commun., Sep./Oct. 2001, vol. 1, pp. $155-158$.

[3] M. L. Morris and M. A. Jensen, "Impact of supergain in multi-antenna systems," in Proc. IEEE Antennas Propagat. Society Intl. Symp., July 2005, vol. 3B, pp. 430-433.

[4] M. L. Morris, M. A. Jensen, and J. W. Wallace, "Superdirectivity in MIMO systems," IEEE Trans. Antennas Propagat., vol. 53, pp. 28502857, Sep. 2005.

[5] M. Uzsoky and L. Solymar, "Theory of super-directive linear antennas," Acta Physica Hungarica, vol. 6, no. 2, pp. 185-205, 1956.

[6] R. F. Harrington, "Antenna excitation for maximum gain," IEEE Trans. Antennas Propagat., vol. 13, pp. 896-903, Nov. 1965.

[7] Y. T. Lo, S. W. Lee, and Q. H. Lee, "Optimization of directivity and signal-to-noise ratio of an arbitrary antenna array," Proc. IEEE, vol. 54, pp. 1033-1045, Aug. 1966.

[8] S. M. Sanzgiri and J. K. Butler, "Constrained optimization of the performance indices of arbitrary array antennas," IEEE Trans. Antennas Propagat., vol. 19, pp. 493-498, July 1971.

[9] L. P. Winkler and M. Schwartz, "A fast numerical method for determining the optimum SNR of an array subject to a Q factor constraint," IEEE Trans. Antennas Propagat., vol. 20, pp. 503-505, July 1972.

[10] N. Yaru, "A note on super-gain antenna arrays," Proc. IRE, vol. 39, pp. 1081-1085, Sep. 1951.

[11] M. L. Morris and M. A. Jensen, "Network model for MIMO systems with coupled antennas and noisy amplifiers," IEEE Trans. Antennas Propagat., vol. 53, pp. 545-552, Jan. 2005.

[12] G. G. Raleigh and J. M. Cioffi, "Spatio-temporal coding for wireless communication," IEEE Trans. Commun., vol. 46, pp. 357-366, Mar 1998.

[13] C. A. Balanis, Antenna Theory: Analysis and Design. New York: Wiley, 1997.

[14] J. Engberg and T. Larsen, Noise Theory of Linear and Nonlinear Circuits. New York: John Wiley \& Sons, 1995.

[15] J. W. Wallace and M. A. Jensen, "Modeling the indoor MIMO wireless channel," IEEE Trans. Antennas Propagat., vol. 50, pp. 591-599, May 2002.

[16] G. J. Burke and A. J. Poggio, "Numerical electromagnetics code (NEC)- method of moments," Lawrence Livermore Laboratory, Tech. Rep. NOSC Tech. Document 116, Jan. 1981.

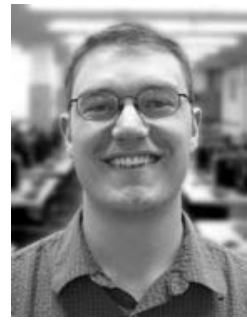

Nicolas W. Bikhazi received the B.S. and Ph.D. degrees in electrical engineering from Brigham Young University (BYU) in 2003 and 2006 respectively.

In 2006 he began working for Sandia National Laboratories. From 2003 to 2006 he was a research assistant at BYU. For the summers of 2001 and 2002 he was an intern at Hewlett-Packard. His research interests are in wireless and optical MIMO communication systems.

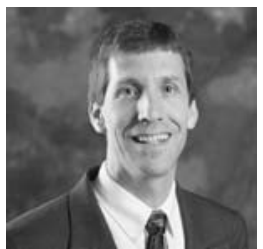

Michael A. Jensen (S'93-M'95-SM'01) received the B.S.(summa cum laude) and M.S. degrees in Electrical Engineering from Brigham Young University (BYU) in 1990 and 1991, respectively, and the $\mathrm{Ph} . \mathrm{D}$. in Electrical Engineering at the University of California, Los Angeles (UCLA) in 1994. From 1989 to 1991 he was a graduate research assistant in the Lasers and Optics Laboratory at BYU. In 1990 he received a National Science Foundation Graduate Fellowship. From 1991 to 1994, he was a graduate student researcher in the Antenna Laboratory at UCLA. Since 1994, he has been at the Electrical and Computer Engineering Department at BYU where he is currently a Professor and Department Chair. His main research interests include antennas and propagation for personal communications, microwave circuit design, radar remote sensing, numerical electromagnetics, and optical fiber communications. He is a member of Eta Kappa Nu and Tau Beta Pi.

Dr. Jensen currently serves as a member of the Administrative Committee and the Joint Meetings Committee for the IEEE Antennas and Propagation Society and as an associate editor for the IEEE TRANSACTIONS ON ANTENNAS AND PROPAGATION. He has also served the society as Vice-Chair and Technical Program Chair for several symposia. He was awarded the $\mathrm{H}$. A. Wheeler paper award in the IEEE TRANSACTIONS ON ANTENNAS AND PROPAGATION in 2002, and the best student paper award at the 1994 IEEE International Symposium on Antennas and Propagation. 